\title{
Application of Terahertz Pulse Time-Domain Holography for Phase Imaging
}

\author{
Nikolay V. Petrov, Maxim S. Kulya, Anton N. Tsypkin, Victor G. Bespalov, and Andrei Gorodetsky
}

\begin{abstract}
Terahertz pulse time-domain holography (THz PTDH) is the powerful technique for high-resolution amplitude and phase THz imaging that allows mapping spectroscopic information across the imaged object. In this paper, we consider most sought after applications of phase imaging provided by this technique and experimentally demonstrate the ability of the method to reconstruct smooth and stepped relief features of an object that is transparent in $\mathrm{THz}$ region. Unlike the amplitude distribution, which does not contain any significant information in this case, phase distribution not only reveals the object qualitatively, but also allows the reconstruction of the object thicknesses pattern, even in low signal-to-noise registration conditions. Main limitations of the proposed method, such as transverse resolution and low signal-to-noise environment are carefully studied and mitigated.
\end{abstract}

Index Terms-Inverse diffraction problem, noise, nondestructive testing, phase retrieval, terahertz holography, terahertz imaging, terahertz radiation.

\section{INTRODUCTION}

$\mathbf{T}$ ERAHERTZ $(\mathrm{THz})$ radiation, which is tightly seated between "classical" microwave and "quantum" optical bands of electromagnetic spectrum, has developed into a separate research field, demanding a unique approach to all stages of implementation, including sources, receivers, and all of the optical components between them, as well as new techniques and algorithms. THz imaging is one of the main applications of this emerging field, especially because most dielectric materials, which are opaque in optical and IR bands, are transparent for $\mathrm{THz}$ waves, and at the same time the wavelength is sufficiently small to achieve some reasonable resolution if compared with microwaves. There are several reviews describing the diversity of approaches and methods of

Manuscript received November 28, 2015; revised January 23, 2016; accepted February 03, 2016. Date of publication April 05, 2016; date of current version June 14, 2016. The work of N. V. Petrov was supported by the Russian Ministry of Education and Science project within the state mission for institutions of higher education under Agreement 2014/190. The work of M. S. Kulya and V. G. Bespalov was supported by the Ministry of Education and Science of the Russian Federation under Grant 3.1675.2014/K. The work of A. N. Tsypkin was supported by the Government of Russian Federation under Grant 074-U01. (Corresponding author: Nikolay V. Petrov.)

N. V. Petrov, M. S. Kulya, A. N. Tsypkin, and V. G. Bespalov are with the Department of Photonics and Optical Informatics, National Research University of Information Technologies, Mechanics and Optics, St. Petersburg 199004, Russia (e-mail: n.petrov@niuitmo.ru).

A. Gorodetsky is with the Optoelectronics and Biomedical Photonics Group, Aston Institute of Photonic Technologies, Aston University, Birmingham B4 7ET, U.K. (e-mail: a.gorodetsky@aston.ac.uk).

Color versions of one or more of the figures in this paper are available online at http://ieeexplore.ieee.org.

Digital Object Identifier 10.1109/TTHZ.2016.2530938
THz imaging [1]-[5]. All imaging techniques can be considered depending on the type of radiation used: continuous wave (CW) and pulsed THz time-domain techniques, because they involve completely different approaches. As described in [5], focused on the comparison between them, $\mathrm{CW} \mathrm{THz}$ imaging allows a more compact and simple transceiver system; phase information, however, due to incoherent registration of the $\mathrm{THz}$ signal in this layout, can be obtained only by the interferometric [6] or holographic [7] approach, which requires a finely aligned and rather complex setup. Moreover, use of monochromatic $\mathrm{CW} \mathrm{THz}$ radiation cannot provide information on wavelength dependence of optical parameters of the sample. In contrary, broadband pulsed $\mathrm{THz}$ radiation generated by means of ultrashort optical laser pulses contains spectral information and allows coherent time-resolved detection.

Among all existing pulsed $\mathrm{THz}$ imaging techniques, the particular niche is occupied by methods based on the standard $\mathrm{THz}$ time-domain spectroscopy (THz-TDS) [8], and one of the reasons for this is the possibility to provide information on materials' spectral features in $\mathrm{THz}$ region [9]. However, despite the fact that they allow obtaining phase reconstruction, most widespread raster scanning THz-TDS systems suffer from restricted lateral resolution determined by the transverse size of the $\mathrm{THz}$ focused waist. This size is defined by several parameters, such as $\mathrm{THz}$ transceiver size and optics used for beam focusing. However, it cannot be squeezed down below the diffraction limit, which is equal to $\sim 0.7 \mathrm{~mm}$ for the frequency of $1 \mathrm{THz}[10]$ without significant power loss, especially in lower frequency components. Some imaging layouts allowed better resolution via integration of signal from several spectral components in reflective time-domain measurement [11], [12]. However, such imaging lacks spectral information. In the latter, detection is performed by matrix CCD camera, and the object image is transferred onto an electrooptic crystal using TPX lens.

Holographic $\mathrm{THz}$ imaging has developed quickly in the last couple of years, becoming more and more actual and mature as a result of the progress in novel compact and powerful THz sources [13]-[16] and THz-capable array detectors [17], [18]. Holographic imaging outperforms both TDS scan and tomographic techniques in acquisition time, provides better spatial resolution, and needs less computational powers for numerical reconstruction than tomography. Recent holographic works demonstrated real-time operation [19], [20] and live reconstruction of moving objects, its applicability for effective biomedical imaging [21], as well as the ability to retrieve both absorptive and phase-shifting images of a sample from a single hologram [22]. However, most of the works have been done with monochromatic CW THz sources [7], [19]-[27] that 
do not provide spectral information, but are reasonably more powerful. Holograms obtained with pulsed $\mathrm{THz}$ setups and coherent wavefront detection has also been reported, but only reconstruction of nontransparent binary objects [28]-[30] has been shown experimentally, despite in-line pulsed holographic approach allowing direct wavefront reconstruction, and both amplitude and phase spatial distribution retrieval has been predicted theoretically [31]. THz pulse time-domain holography (PTDH) offers several significant advantages over TDS: 1) PTDH is realized in a collimated beam, which means that the object can be stationary placed at any position inside it, which is often needed in real-life measurements; 2) it is practically optics-free, after the beam is collimated, it goes straight to the detection stage, and the reconstruction quality does not depend on optics used in the layout; 3 ) it provides significantly faster detection, with only a temporal scan needed [28]; and 4), most importantly, the achievable resolution, which is of the order of one wavelength [30], is significantly higher, because in PTDH layout one is not limited by the beam waist size as in TDS [32]. Furthermore, the resolution in PTDH can be even improved by sacrificing the spectral information and incorporation of multiple wavelengths to achieve subwavelength resolution [8], [12] that outperforms even CW registration schemes [22]. Z-resolution of PTDH reconstruction of transparent objects surpasses the one obtained with reflective imaging, where it is restricted by coherence length of the radiation [3], due to the $\mathrm{THz}$ pulse overlapping problem [12]. PTDH transmission scheme helps to avoid this problem, because the transmitted pulse is measured, and resolution in the $z$-direction is defined by the duration of femtosecond probe pulses and the precision of the optical delay line, as in a TDS scan. Thus, THz PTDH is the ultimate layout for imaging of amplitude and phase objects that provides the best uncompromising resolution in all directions among all far-field imaging techniques, as well as spectral information. However, the lack of compact and yet powerful pulsed $\mathrm{THz}$ sources results in the fact that noise becomes a serious issue in coherent measurement. To resolve this problem, an effect of noise should be carefully studied to properly choose between existing noise-canceling methods and adapt them for PTDH.

In this paper, we consider the range of unique features of THz PTDH and define four classes of problems related with phase imaging that are associated with sought-after applications. Among these tasks, we focused on the reconstruction of smooth and stepped relief features of an object that is transparent for $\mathrm{THz}$ radiation. We experimentally demonstrate advantages of phase imaging over amplitude imaging, incapable of resolving complex irregular object topology. Also, we study numerically the effect of noise on the reconstruction to show the principal possibility to derive the wavefront even in the cases where signal and noise are comparable.

This paper is organized as follows. Section II presents the theoretical description of the THz PDTH method, explains the experimental setup to measure temporal waveforms of $\mathrm{THz}$ diffracted waves, and presents a mathematical model to reconstruct amplitude and phase characteristics of an object. The discussion of phase-imaging tasks that can be solved by the method are also given in this section. The description of performed

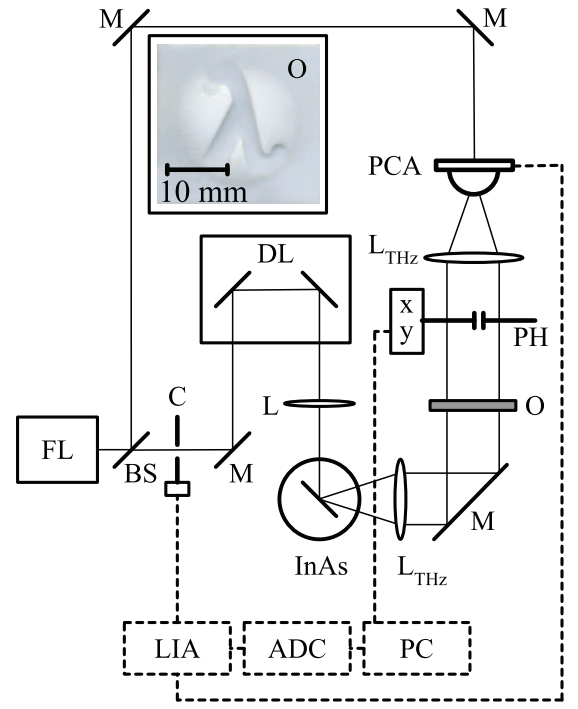

Fig. 1. Setup layout. FL: femtosecond laser ( $\tau=20 \mathrm{fs}, \lambda=790 \mathrm{~nm}$, repetition rate $70 \mathrm{MHz}, P=300 \mathrm{~mW}$ ); BS: beam splitter; C: mechanical chopper; DL: delay line; InAs: THz generator; M: mirrors; L: lens; O: Sample; PH: pinhole; x-y: 2-D scan stage; $L_{\mathrm{THz}}$ : lens for THz; PCA: photoconductive antenna; LIA: lock-in amplifier: ADC: digitizer.

simulations and their outcome with discussion is presented in Section III. The experimental validation of the presented claims can be found in Section IV. In Section V, all results are listed with conclusions and foresights of further steps to be done to advance PTDH for real-life applications.

\section{BACKGROUND AND THEORY}

\section{A. Experimental Setup}

The main schematics feature of $\mathrm{THz}$ PTDH involves measurement of diffraction pattern of the collimated $\mathrm{THz}$ pulse field spatial distribution at some distance behind the object in the time-domain regime, thus allowing reconstruction of amplitude and phase distribution at the object plane by numerical backpropagation of the wavefront in the spectral domain.

The setup is shown in Fig. 1.

The beam of the femtosecond laser (pulse duration $\tau$ $=20 \mathrm{fs}$, central wavelength $\lambda=790 \mathrm{~nm}$, repetition rate $70 \mathrm{MHz}$, mean power $300 \mathrm{~mW}$ ) is split into pump and probe beams of equal power. The probe beam runs directly to a photoconductive antenna (BATOP iPCA) that is used for coherent detection. The pump beam passes through the delay line to fulfil classic time-domain coherent detection and then to the $\mathrm{THz}$ generator. We use InAs crystal placed into a permanent $\mathrm{NdBFe}$ magnet system providing a magnetic field of $2.4 \mathrm{~T}$ at the crystal surface for optical-to-THz conversion enhancement [33]. The produced $\mathrm{THz}$ radiation is collimated for the measurement stage and then focused onto a photoconductive antenna for time-domain detection [34] by a terahertz TPX 2-in lens (Tydex Ltd.) with a focal distance of $50 \mathrm{~mm}$. A mechanical chopper modulates the THz pump beam to allow lock-in detection for the signal-to-noise ratio (SNR) increase. Using a motorized delay line, we change the overlap of the $\mathrm{THz}$ and optical pulses on the antenna to obtain the $\mathrm{THz}$ pulse time-domain profile. Since currently we do not possess either large ZnTe crystal 
[28], [35], [36] or other coherent array $\mathrm{THz}$ detectors, we recorded the wavefront by moving a pinhole aperture placed $5 \mathrm{~mm}$ behind the object and immediately before the TPX lens inside the collimated beam. Such an approach has already been shown previously [37], even though it exhibits faster fall-off of the registered signal when moving from the center to the edge due to the change of the angle between the signal wave vector and optical axis, and the projection of the signal is proportional to the cosine of this angle. Measured voltage 3-D space-time dataset is then numerically processed as described below to reconstruct the $\mathrm{THz}$ wavefront.

\section{B. Mathematical Model of THz PTDH}

Using the experimental layout registration technique mentioned above, we obtain the $\mathrm{THz}$ pulse time-domain profiles $E_{x, y}(t)$ for every position of the pinhole on an $(x-y)$ motorized stage within the collimated beam. Then, the time-profiles are converted to obtain a $\mathrm{THz}$ spectra at every point of the wavefront:

$$
U_{x, y}(\nu)=\mathcal{F}_{1 D}\left(E_{x, y}(t)\right)=\int_{0}^{\infty} E_{x, y}(t) \exp (-2 \pi i \nu) d t .
$$

$\mathcal{F}_{1 D}$ denotes 1-D Fourier transform. These spectra are complex numbers $U(x, y, \nu)=|U(x, y, \nu)| \exp (i \varphi(x, y, \nu))$ that contain both amplitude $|U(x, y, \nu)|$ and phase $\varphi(x, y, \nu)$ spatial distribution. To reconstruct amplitude and phase wavefront perturbations caused by the object, this field should be numerically propagated to the object plane. For this purpose, scalar diffraction theory [38] can be used.

The field at the registration plane is formed as follows. Let $u\left(x^{\prime}, y^{\prime}, \nu\right)$ be the spectro-spatial field distribution at the object plane, and $\mathrm{F}_{l, \nu}$ is the operator of the broadband THz field propagation from the object plane $\left(x^{\prime}, y^{\prime}\right)$ to the registration plane $(x, y)$ as follows:

$$
U(x, y, \nu)=\mathrm{F}_{l, \nu}\left[u\left(x^{\prime}, y^{\prime}, \nu\right)\right] .
$$

More detailed description of propagators used for reconstruction in PTDH method involving broadband THz two-dimensional field forward propagation can be found in [38] and partly in [27], [39], [40], for monochromatic waves.

For backward propagation of the single frequency wavefront component from the registration plane to the object plane, within scalar diffraction theory approach, the inverse operator $\mathrm{F}_{l, \nu}{ }^{-1}$ should be used. As operator $\mathrm{F}_{l, \nu}{ }^{-1}$, the two most common methods of wavefront propagation can be used: angular spectrum method (ASM) and Rayleigh-Sommerfeld convolution (RSC). They calculate wavefront from the hologram plane $(x, y)$ to the object plane $\left(x^{\prime}, y^{\prime}\right)$ and one should bear in mind that their applicability is limited by the sampling theorem. Thus, ASM method is defined as

$$
\begin{aligned}
u\left(x^{\prime}, y^{\prime}, 0\right)= & \iint_{-\infty}^{+\infty} U\left(f_{x}, f_{y}\right) \exp \left(2 \pi i\left(x^{\prime} f_{x}+y^{\prime} f_{y}\right)\right) \\
& \times \exp \left(-i 2 \pi \sqrt{\frac{\nu^{2}}{c^{2}}-\left(f_{x}{ }^{2}+f_{y}{ }^{2}\right)}\right) d f_{x} d f_{y}
\end{aligned}
$$

in which

$$
\begin{aligned}
U\left(f_{x}, f_{y}\right)= & \iint_{-\infty}^{+\infty} \exp \left(-i 2 \pi\left(x f_{x}+y f_{y}\right)\right) \\
& \times U(x, y, l) d x d y
\end{aligned}
$$

is the angular spectrum in spatial frequencies $\left(f_{x}, f_{y}\right)$. If $\Delta x=$ $\Delta y$, the method is restricted by the inequality [39]

$$
\nu \geq \nu_{0}=\frac{c \cdot l}{D \cdot \Delta x}
$$

that defines the frequencies $\nu$ in the $\mathrm{THz}$ spectrum that can be used for reconstruction of the object inline hologram recorded at the distance $l$ by the detector of the size $D$ and pixel size $\Delta x$. $c$ denotes the speed of light.

Similarly, the field $u\left(x^{\prime}, y^{\prime}, 0\right)$ for frequencies $\nu \leq \nu_{0}$ can be calculated using the RSC method

$$
u\left(x^{\prime}, y^{\prime}, 0\right)=U(x, y, l) * h^{-1}\left(x^{\prime}, y^{\prime}, 0\right)
$$

where $h^{-1}$ is the impulse response function

$$
h^{-1}\left(x^{\prime}, y^{\prime}, 0\right)=\frac{\nu \exp \left(-i 2 \pi r \nu c^{-1}\right)}{i \cdot c \cdot r} \frac{l}{r}
$$

and $r=\left(l^{2}+\left(x-x^{\prime}\right)^{2}+\left(y-y^{\prime}\right)^{2}\right)^{1 / 2}$ is the distance between the object and registration planes.

Thus, spectral components of the broadband $\mathrm{THz}$ spectrum in PTDH should be propagated forward and backward by $F_{l, \nu}$ and $F_{l, \nu}{ }^{-1}$ equal to either (3) or (6), depending on whether the component frequency is above or below $\nu_{0}$, correspondingly.

\section{THz PTDH Applications}

Samples of low-absorption material often do not introduce sufficient amplitude change to the wavefront to be characterized by recovered amplitude, but, having at hand both amplitude and phase information of the inverted broadband wavefront, we can get the transparent object characteristics. THz PTDH can be used for solving four different tasks, depending on preliminary object information.

i) If optical characteristics of the sample material in the $\mathrm{THz}$ range are known, its thickness profile can be derived from phase difference of reconstructed background and hologram wavefronts as

$$
\Delta z\left(x^{\prime}, y^{\prime}\right)=\frac{\Delta \varphi\left(x^{\prime}, y^{\prime}\right) c}{2 \pi \nu(n(\nu)-1)}
$$

where $n(\nu)$ is the refractive index value at frequency $\nu$ and $\Delta \varphi\left(x^{\prime}, y^{\prime}\right)$ is the frequency-domain unwrapped phase difference between background and hologram fields at the object plane.

Use of broadband spectrum for thickness reconstruction (8) allows getting better lateral and longitudinal resolution. The object relief for a known material can be obtained as

$$
\Delta z\left(x^{\prime}, y^{\prime}\right)=\frac{1}{M} \sum_{m=1}^{M} \frac{\Delta \varphi\left(x^{\prime}, y^{\prime}\right) c}{2 \pi \nu_{m}\left(n\left(\nu_{m}\right)-1\right)} .
$$


ii) If a sample profile is measured by any other profilometric technique, material optical characteristics in the $\mathrm{THz}$ range can be derived for every point of the sample as

$$
\begin{aligned}
n\left(x^{\prime}, y^{\prime}, \nu\right)= & \frac{\Delta \varphi\left(x^{\prime}, y^{\prime}\right) c}{2 \pi \nu \Delta z}+1 \\
k\left(x^{\prime}, y^{\prime}, \nu\right)= & -\frac{c}{2 \pi \nu \Delta z} \times \\
& \times \ln \left(\frac{A_{H}\left(x^{\prime}, y^{\prime}\right)}{A_{B}\left(x^{\prime}, y^{\prime}\right)} \frac{\left(n\left(x^{\prime}, y^{\prime}, \nu\right)-1\right)^{2}}{4 n\left(x^{\prime}, y^{\prime}, \nu\right)}\right)
\end{aligned}
$$

where $n\left(x^{\prime}, y^{\prime}, \nu\right)$ is the refractive index for the frequency $\nu$ at the point with coordinates $\left(x^{\prime}, y^{\prime}\right), k\left(x^{\prime}, y^{\prime}, \nu\right)$ is the extinction coefficient at the same point, and $A_{H}\left(x^{\prime}, y^{\prime}\right)$ and $A_{B}\left(x^{\prime}, y^{\prime}\right)$ are the amplitudes of hologram and background reconstructed wavefronts, correspondingly.

iii) If it is supposed that the object contains several materials featuring strongly distinguished differences in their $\mathrm{THz}$ absorption lines (e.g., explosives [41] or drugs [42]), THz PTDH will allow determining the lateral distribution of all concealed matter within the sample. It is possible because thickness derivation from the recovered wavefront at different frequencies will show discrepancies only at characteristic spectral lines where the odd material is located in the object. Thus, not only the position of the concealed matter can be determined, but also the matter itself can be divined.

iv) If object material is unknown, its characteristics and thickness still can be recovered, however this will require a significant upgrade of the experimental setup to enable transflective measurement analogous to transflective digital holographic microscopy in visible range [43]. Such a setup has been implemented for TDS [44], but its advance for full transmitted and reflected wavefronts in wide-beam layout registration without serious loss of SNR will be the next big challenge for $\mathrm{THz}$ imaging which will allow solving new and vital tasks, e.g., reconstruction of 3-D material distribution of hidden objects.

\section{NumericAl SimUlation}

We have already modeled the process of recording holograms of amplitude and phase objects and their further successful reconstruction to show fundamental applicability of THz PTDH for imaging, to analyze its resolving power, and to find optimal layout for experiment realization in our previous works [30], [31], [38], [39], [45]-[49]. Here, we perform numerical simulation of the experiment for the setup depicted in Fig. 1 to confirm the conceptual applicability of the reconstruction method in the case of a relatively low SNR environment that we could achieve in our setup when scanning with a pinhole of a small diameter.

In any measurement, two distinct noises should be taken into account: incoming emitter noise $\Theta^{(e)}(t)$ and detector noise $\Theta^{(d)}(t) . \Theta^{(e)}$ is usually proportional to signal and can be estimated by averaging several time-domain scans and deriving their standard deviation $\sigma: \Theta^{(e)}(t) \sim \sigma(E(t))[50]$. $\Theta^{(d)}(t)$ can be obtained by measuring the signal from the detector with $\mathrm{THz}$ path blocked, i.e., without any actual coherent $\mathrm{THz}$ wave falling onto it, this noise does not depend on signal amplitude, but has its own spectrum. Usually, in finely aligned TDS setup, when the considerable part of the generated $\mathrm{THz}$ pulse reaches the detector, $\Theta^{(d)}(t)$ is much (more then three orders of magnitude) smaller than $\Theta^{(e)}(t)$. By introducing a pinhole into the broad collimated beam, we block the major part of the wavefront energy, and the smaller the pinhole area is, the less signal reaches the detector.

Thus, detector-dependent $\Theta^{(d)}(t)$, that remains unchanged becomes comparable to $\Theta^{(e)}(t)$ and even the signal itself, thus leading to an SNR drop in the measured signal $\tilde{E}_{x, y}=E_{x, y}(t)+\Theta_{x, y}^{(e)}(t)+\Theta_{x, y}^{(d)}(t)$.

On the other hand, by decreasing pinhole aperture, we obtain better spatial resolution in the hologram, and the problem of optimal pinhole size should be solved before running the experiment.

Basically, what we get from the measurement as an input for reconstruction is

$$
\tilde{U}_{x, y}(\nu)=\hat{\mathcal{F}}_{1 D}\left(E_{x, y}(\nu)+\Theta_{x, y}^{(e)}(\nu)+\Theta_{x, y}^{(d)}(\nu)\right) .
$$

To simulate the effect of the noise, we measured $\Theta^{(d)}(t)$ amplitude spectrum and ratio $\kappa=\left|\Theta^{(e)}(t)\right| /|E(t)|$ for our setup. Then, after simulation of forward propagation of the wavefront, diffracted at the phase object, similar to the one that was prepared for the experiment, measured $\Theta^{(d)}(t)$ and $\Theta^{(e)}(t)$ at the level $\kappa$, were added to it at every point of the hologram. This noise-disturbed wavefront was back-propagated to reconstruct the object.

We simulate backward wavefront propagation for holograms recorded with pixels of different area to estimate the optimal pinhole size for the best object reconstruction quality. By making the pixel size smaller (bigger), we get higher (lower) reconstruction resolution and contrast, and, at the same time, lower (higher) SNR correspondingly, because $E(t)$ is proportional to the pinhole area, but $\Theta^{(d)}(t)$ does not change. In our simulations we set the distance between target and hologram to be $5 \mathrm{~mm}$ because at this distance Fresnel numbers $F_{m}^{\#}=D^{2} \nu_{m} / 4 c l$ for this configuration lie in the range $10<F_{m}^{\#}<50$ for all frequencies $\nu_{m}$ used. These values correspond to the optimal reconstruction conditions [46], [49].

Phase object used in the simulations is shown in Fig. 2(a); it introduces phase shift that is proportional to the frequency $\Delta \varphi=2 \pi \Delta z \nu n c^{-1}$ and no amplitude change. Broadband intensity at the hologram plane is shown in Fig. 2(b). Spectra corresponding to the pinholes with different sizes is plotted in Fig. 2(c). In Fig. 2(d), each row shows pinhole size used for simulated hologram registration. Simulated time-domain profiles at two positions of the pinhole on the generated hologram one at very central and one at very utmost point, with $\Theta^{(e)}(t)$ and $\Theta^{(d)}(t)$ added numerically for all pinhole sizes from Fig. 2(d) are plotted in Fig. 2(e). Corresponding points are shown in Fig. 2(b) by blue (center) and red (edge) arrows. In Fig. 2(f) and (g), object reconstructions, simulated from noisy holograms using spectral components of 0.3 and $0.4 \mathrm{THz}$, respectively, are shown. These spectral components were chosen because they are close to the spectral maximum of the signal we use. As can be seen from Fig. 2(f) and (g), for all pinhole sizes and hence SNRs simulated, the object still can be recognized, even for pinhole sizes of 0.5 and $0.4 \mathrm{~mm}$, when 
(a)

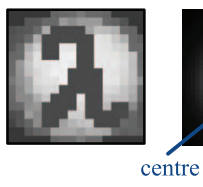

(d) (b)

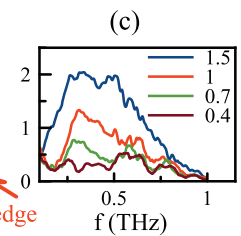

(f)

(g)

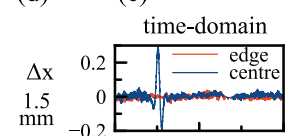

$\mathrm{mm}$
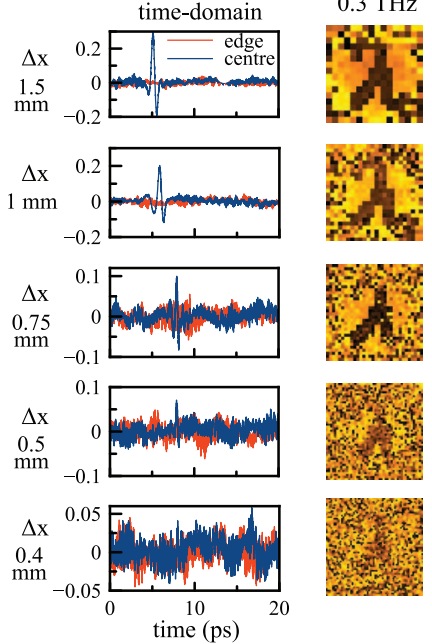

$0.4 \mathrm{THz}$
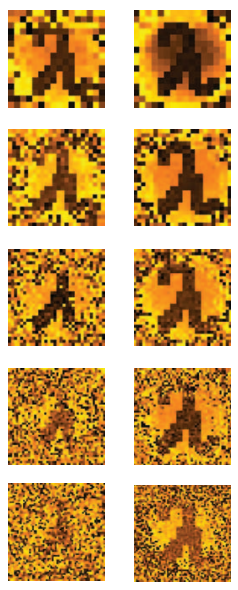

Fig. 2. (a) Initial phase object, corresponding to one used in the experiment. (b) Simulated broadband hologram intensity. (c) Spectra corresponding to the pixels of different sizes. (d) Pixel sizes. (e) Time-domain profiles at hologram plane in the center and at the edge of it, and object reconstructions with (f) 0.3 and $(\mathrm{g}) 0.4 \mathrm{THz}$, respectively, for different pinhole sizes.

$E(t) \sim \Theta^{(d)}(t)$. To estimate reconstruction quality, we use normalized absolute error, defined as [51]

$$
\mathrm{NAE}=\frac{\sum_{i} \sum_{j}\left|u\left(x_{i}, y_{j}\right)-\tilde{u}\left(x_{i}, y_{j}\right)\right|}{\sum_{i} \sum_{j}\left|u\left(x_{i}, y_{j}\right)\right|} .
$$

NAE dependencies on frequency and SNR are shown in Fig. 3. Here, the percents show the ratio between the noise and signal amplitudes multiplied by $100 \%$. For all traces, opposed noise was kept at $10 \%$ for $\Theta^{(d)}(t)$ and $0 \%$ for $\Theta^{(e)}(t)$. Fall of reconstruction quality for lower frequency components $(\leq 0.3 \mathrm{THz})$ is mostly caused by loss of resolution [30] and hardly affected by noise, because signal in this spectral region is relatively high (see Fig. 2(c)). However, signal at higher frequency components $(\geq 0.5 \mathrm{THz})$ that provide better resolution and contrast, is significantly lower. Hence reconstruction result is more affected by noise, thus leading to losses in quality, but due to a different reason. Hence, only frequencies between 0.3 and $0.5 \mathrm{THz}$ can be used for reconstruction, despite the fact that the signal is higher at frequencies below and resolution is better above. As can be seen from Fig. 2, among the tested scanning apertures, a pinhole of $1.5 \mathrm{~mm}$ in diameter can be used for hologram recording, since it still allows recovery of all object features and at the same time gives the highest SNR. Further numerical noise canceling techniques can be used to achieve even better reconstruction quality [52].

\section{EXPERIMENTAL RESUlts}

Previously, THz PTDH has been shown experimentally to reconstruct amplitude objects only [30]. In this paper, we focus on (a)

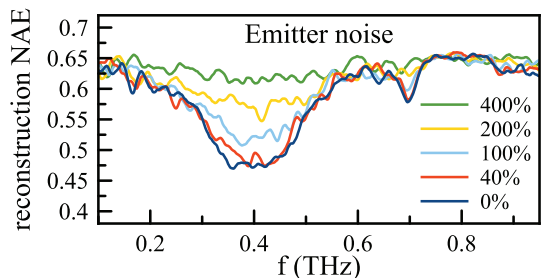

(b)

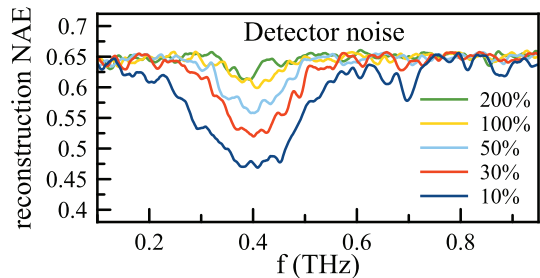

(c)

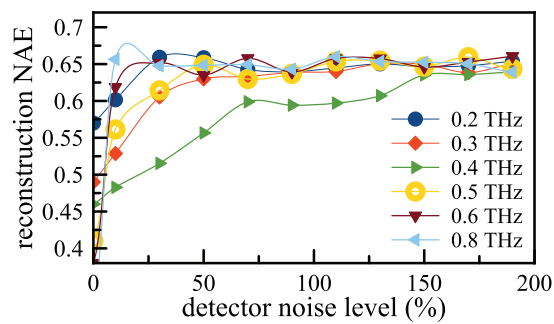

Fig. 3. (a) NAE of reconstructed image for different levels of emitter noise. (b) NAE of reconstructed image for different levels of detector noise. (c) NAE of the image reconstructed with different wavelengths versus detector noise level.

experimental reconstruction of the phase object by THz PTDH. Since we know the optical properties of the material, we solve the task i) from four described in Section II-C.

To get the wavefront spatial distribution, we scan the broad collimated beam with a pinhole [37] of $1.5-\mathrm{mm}$ diameter at the distance of $5 \mathrm{~mm}$ from the object. The $\mathrm{THz}$ beam waist allows scanning the area of $24 \times 24 \mathrm{~mm}$, i.e., Fresnel number for this configuration is $F^{\#}=D^{2} \nu / 4 c l \approx 30$ at $\nu=0.3 \mathrm{THz}$. To get more information for reconstruction, we make $32 \times 32$ temporal scans within this area.

In our experiment, we used a Teflon test object shown in the inset of Fig. 1. It is transparent for $\mathrm{THz}$ radiation and has the shape of the $\lambda$ letter cut in a spherical surface of the Teflon object with equipment provided by Fablab Polytech. Thus, the object featured both smooth and steep profile changes. The circle diameter is $21 \mathrm{~mm}$, and the maximum cut depth is $1.5 \pm 0.1 \mathrm{~mm}$.

To get object thickness, we measured the refractive index of the Teflon at $\mathrm{THz}$ frequencies using a different flat piece of material and standard THz TDS setup (of the same emitter and detector) with focused beam. The spectrum that can be measured on our system lies within $0.05-1.3 \mathrm{THz}$ [53], with the spectral resolution of $10 \mathrm{GHz}$. The SNR at maximum is 250 .

As one can see from Fig. 4(c), the value of refractive index of the Teflon is constant for all frequencies in the measured $\mathrm{THz}$ spectral range and is equal to 1.46 . This result coincides with the value of refractive index mentioned in [54].

To provide careful comparison between measurements and simulation we plot spatio-temporal cross sections of the $\mathrm{THz}$ wavefront with and without the object introduced. Fig. 5 depicts the perfect concordance of the measured spatio-temporal profiles of the undisturbed background THz wavefront [Fig. 5(a) and (c)] with corresponding simulation results [Fig. 5(b) and 

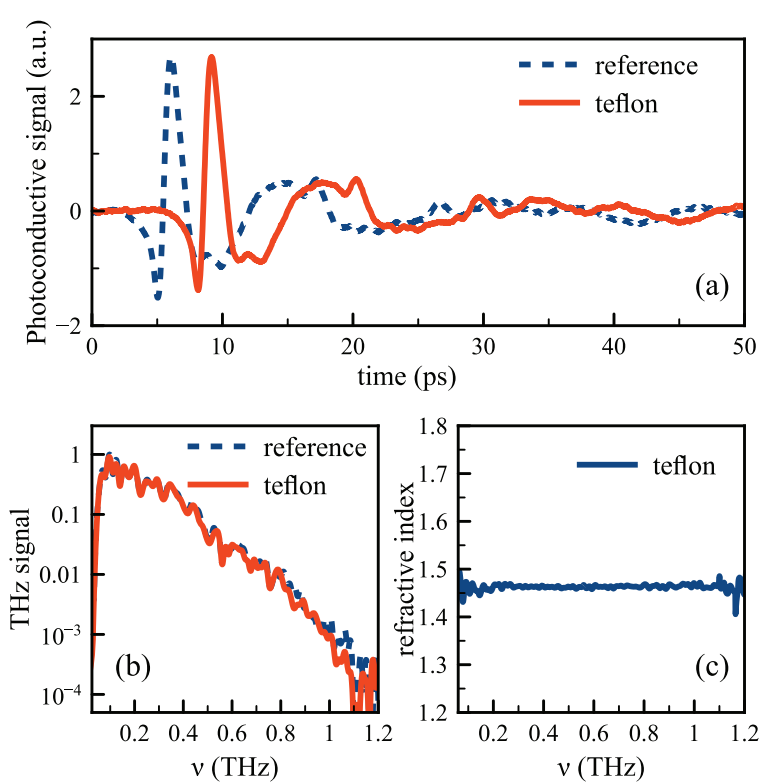

Fig. 4. (a) THz reference (blue dashed) and teflon sample (solid red) time-domain profiles, (b) their corresponding $\mathrm{THz}$ power spectra, and Teflon refractive index for $\mathrm{THz}$ frequencies derived from these measurement.

(d)], and similar results for the wavefront that rubbed through the sample [Fig. 5(e)-(h)]. From the background profile, it can be seen that axial part of $\mathrm{THz}$ wavefront is slightly ahead of peripheral. This field behavior was predicted by the model based on diffraction broadening [55].

Since the sample used in our experiment does not attenuate the amplitude significantly, its relief information is encoded in the spatial distribution of the phase. Even though during its propagation to the registration plane the wavefront has changed, we can notice the shade of the object pattern in the phase spatial distribution of some spectral components in the registration plane (Fig. 6). Amplitude distribution in our experiment does not reveal the object.

As described in Section II-B, the recorded wave field in the registration plane has been propagated to the object plane. Frequency $\nu_{0}$ defined in (5) for our setup is equal to $\nu_{0} \approx 0.08 \mathrm{THz}$, thus, for numerical wavefront back propagation, we used (3). After phase distribution is obtained at object plane both for background and object wavefronts, (8) is used to transform the phase to the object relief.

The reconstructed 2-D object relief profiles obtained from the derivation of data from single monochromatic component of $0.33 \mathrm{THz}$, from a number of components between 0.3 and $0.33 \mathrm{THz}$, and from a components of a more broad spectrum of 0.3 to $0.4 \mathrm{THz}$ are plotted in Fig. 7. Involving the bigger number of spectral components improves object reconstruction, as was mentioned in [12] but only up to some certain limit. By adding the phase information from the lower frequencies, we lose the spatial resolution in all directions, because $x-y$ resolution of reconstruction is defined by the wavelength [30], and $z$-resolution changes linearly with the wavelength. By joining phase distribution data of the higher frequency components, we bring more noise into the image, because the spectrum we have is relatively narrow and SNR vanishes with the frequency growth. (a)

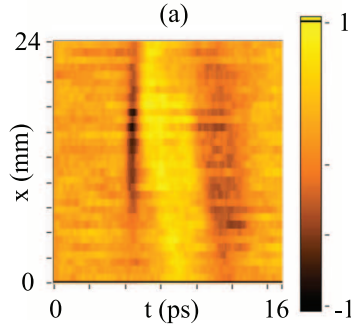

(c)

(e)

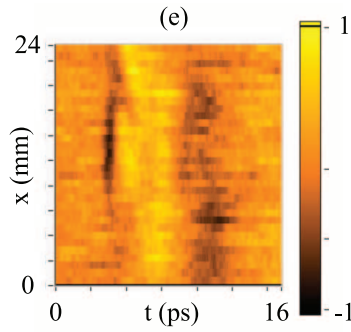

(g)

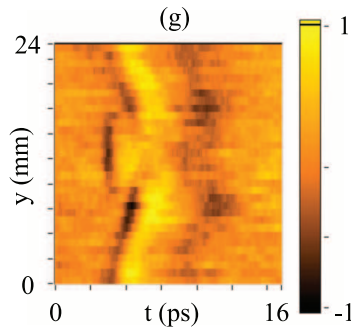

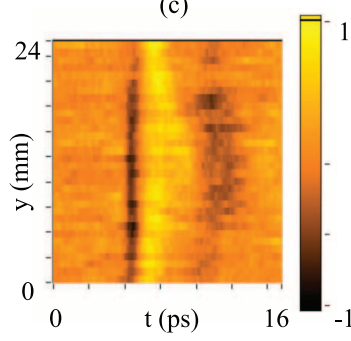

(b)

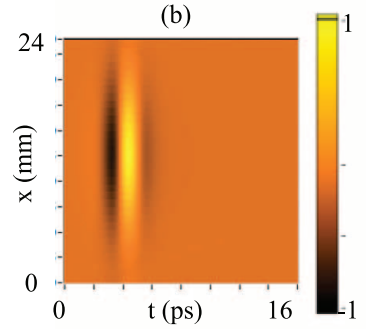

(d)

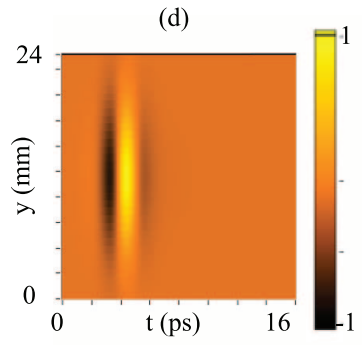

(f)

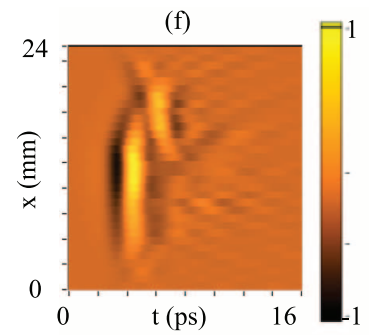

(h)

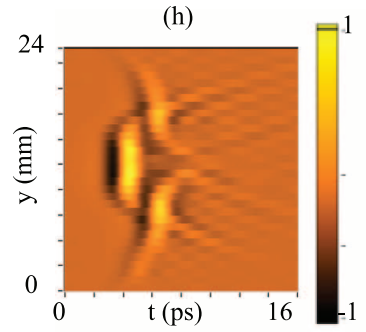

Fig. 5. (a), (c) Measured and (b), (d) simulated spatio-temporal profiles of undisturbed $\mathrm{THz}$ wavefront and $\mathrm{THz}$ wavefront that passed through the sample, $(\mathrm{e}),(\mathrm{g})$ measured and (f), (h) simulated.
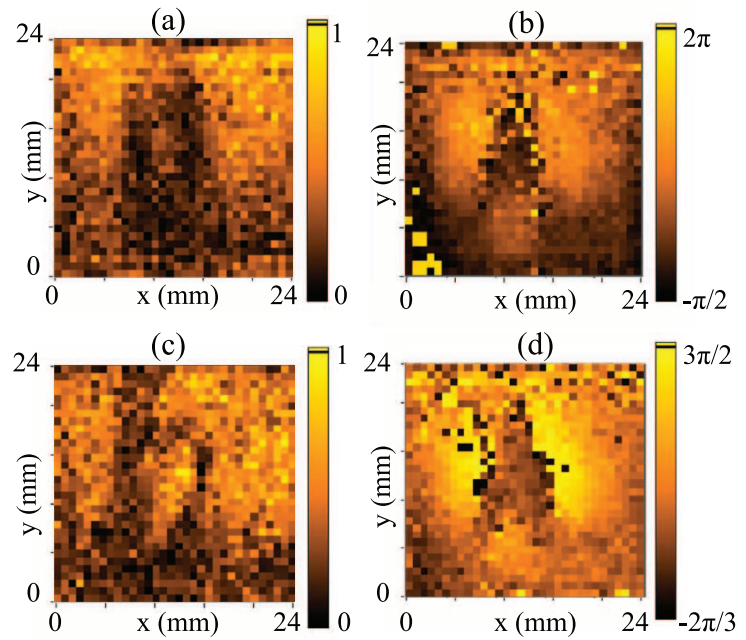

Fig. 6. Spatial distribution of (a), (c) the amplitude and (b), (d) the phase in the registration plane at a frequency of (a), (b) $0.25 \mathrm{THz}$ and (c), (d) $0.35 \mathrm{THz}$. 
(a)
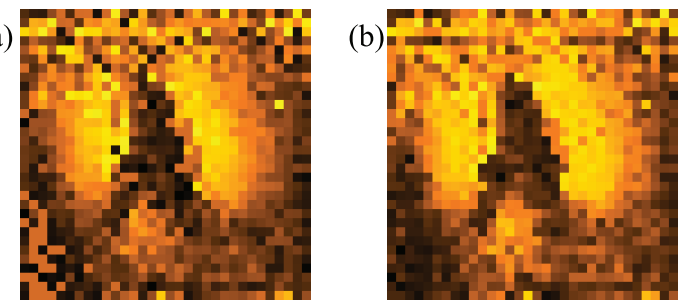

(c)
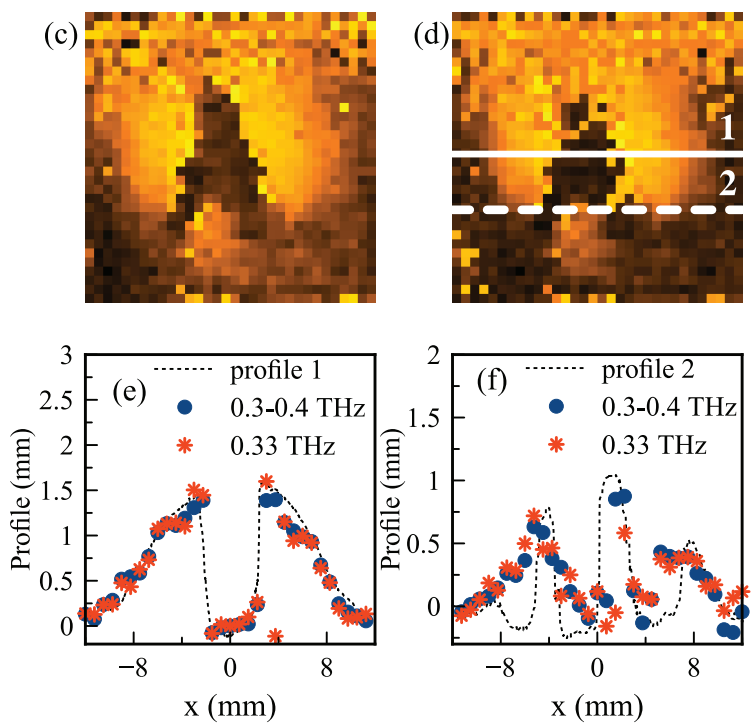

Fig. 7. Object relief reconstructed with (a) single monochromatic component of $0.33 \mathrm{THz}$, (b) with components between 0.3 and $0.33 \mathrm{THz}$, (c) with components between 0.3 and $0.4 \mathrm{THz}$, and (d) components between 0.25 and 0.4 THz. Lines 1 and 2 in (d) show cross section for profiles plotted in (e) and (f), respectively.

Thus, as we already proposed in Section III, both of these limitations should be taken into account and the spectral components that are used for the reconstruction should be chosen only within $0.3-0.5-\mathrm{THz}$ range. To demonstrate the accurateness of the reconstruction, the retrieved thickness is plotted for the two cross sections of the reconstructed object together with the object profile measured by $3 \mathrm{~d}$ laser scanning with CRONOS3D DUAL scanner, the positions of the selected cross sections are shown in Fig. 7(d). From the plots in Fig. 7(e) and (f), the correspondence between reconstructed and actual thickness for both central and side cross-sections is clearly seen.

\section{CONCLUSION}

We have promoted $\mathrm{THz}$ pulse time-domain holography $(\mathrm{THz}$ PTDH) as the perspective method of $\mathrm{THz}$ imaging, compared it with other imaging techniques in this frequency range, including different approaches to holography with $\mathrm{THz}$ waves, and proposed the unique imaging tasks that can be effectively solved by $\mathrm{THz}$ PTDH. For the first time, we experimentally demonstrated the ability of THz PTDH to reconstruct phase objects with smoothly varying thickness as well as stepped features with wavelength lateral and sub-wavelength longitudinal resolution.

The THz PTDH method is based on the full $\mathrm{THz}$ wavefront measurement and subsequent numerical wavefront inversion. Spatial phase distribution in the object plane allows reconstruction of the object relief, even if amplitude distribution does not provide essential information for the object reconstruction and the object is very transparent for $\mathrm{THz}$ radiation.

Incorporation of a larger number of $\mathrm{THz}$ spectral components provides better reconstruction characteristics, but only up to a certain extent, because, by joining phase data of the higher frequency components that are drastically weaker, no significant data is added.

The effect of emitter and detector noise on image reconstruction quality was studied, and reconstruction demonstrated surprisingly very high noise tolerance of the method. In our simulations, even if the noise level was comparable to the signal at hologram, the object was still retrievable.

\section{ACKNOWLEDGMENT}

A. Gorodetsky would like to thank Magicplot Systems, LLC, for providing MagicPlot Pro cross-platform plotting software used for preparation of all figures in the paper. N. V. Petrov would like to thank Fablab Polytech for providing equipment for the test object preparation.

\section{REFERENCES}

[1] D. Mittleman, M. Gupta, R. Neelamani, R. Baraniuk, J. Rudd, and M. Koch, "Recent advances in terahertz imaging," Appl. Phys. B, vol. 68, no. 6, pp. 1085-1094, 1999.

[2] J. P. Guillet, B. Recur, L. Frederique, B. Bousquet, L. Canioni, I. Manek-Hönninger, P. Desbarats, and P. Mounaix, "Review of terahertz tomography techniques," J. Infrared Millim. THz., vol. 35, no. 4, pp. 382-411, 2014.

[3] W. L. Chan, J. Deibel, and D. M. Mittleman, "Imaging with terahertz radiation," Rep. Prog. Phys., vol. 70, no. 8, p. 1325, 2007.

[4] P. U. Jepsen, D. G. Cooke, and M. Koch, "Terahertz spectroscopy and imaging - Modern techniques and applications," Laser Photon. Rev., vol. 5, no. 1, pp. 124-166, 2011.

[5] N. Karpowicz, H. Zhong, J. Xu, K.-I. Lin, J.-S. Hwang, and X. Zhang, "Comparison between pulsed terahertz time-domain imaging and continuous wave terahertz imaging," Semicond. Sci. Technol., vol. 20, no. 7, p. S293, 2005.

[6] J. L. Johnson, T. D. Dorney, and D. M. Mittleman, "Interferometric imaging with terahertz pulses," IEEE J. Sel. Topics Quantum Electron., vol. 7, no. 4, pp. 592-599, Jul-Aug. 2001.

[7] M. S. Heimbeck, M. K. Kim, D. A. Gregory, and H. O. Everitt, "Terahertz digital holography using angular spectrum and dual wavelength reconstruction methods," Opt. Exp., vol. 19, no. 10, pp. 9192-9200, 2011.

[8] L. Zhang, Y. Zhang, C. Zhang, Y. Zhao, and X. Liu, "Terahertz multiwavelength phase imaging without $2 \pi$ ambiguity," Opt. Lett., vol. 31, no. 24, pp. 3668-3670, 2006.

[9] L. Zhang, H. Zhong, C. Deng, C. Zhang, and Y. Zhao, "Terahertz wave reference-free phase imaging for identification of explosives," Appl. Phys. Lett., vol. 92, no. 9, 2008, Art. ID 091117.

[10] M. Herrmann, R. Fukasawa, and O. Morikawa, "Terahertz imaging," in Terahertz Optoelectronics. Berlin, Germany: Springer-Verlag, 2005, vol. 382, pp. 331-382.

[11] T. Buma and T. B. Norris, "Time reversal three-dimensional imaging using single-cycle terahertz pulses," Appl. Phys. Lett., vol. 84, no. 12, pp. 2196-2198, 2004.

[12] L. Zhang et al., "Terahertz wave focal-plane multiwavelength phase imaging," J. Opt. Soc. Amer. A, vol. 26, no. 5, pp. 1187-1190, 2009.

[13] M. A. Belkin and F. Capasso, "New frontiers in quantum cascade lasers: High performance room temperature terahertz sources," Phys. Scripta, vol. 90, no. 11, 2015, Art. ID118002.

[14] M. Razeghi et al., "Quantum cascade lasers: From tool to product," Opt. Exp., vol. 23, no. 7, p. 8462, 2015.

[15] M. Scheller, J. M. Yarborough, J. V. Moloney, M. Fallahi, M. Koch, and S. W. Koch, "Room temperature continuous wave milliwatt terahertz source," Opt. Exp., vol. 18, no. 26, 2010, Art. ID 27112.

[16] C. W. Berry, N. Wang, M. R. Hashemi, M. Unlu, and M. Jarrahi, "Significant performance enhancement in photoconductive terahertz optoelectronics by incorporating plasmonic contact electrodes," Nat. Commun., vol. 4, p. 1622, 2013. 
[17] D.-T. Nguyen, F. Simoens, J.-L. Ouvrier-Buffet, J. Meilhan, and J.-L. Coutaz, "Simulations and measurements of the electromagnetic response of broadband $\mathrm{THz}$ uncooled antenna-coupled microbolometer array," in Proc. 19th Int. Conf. Microw., Radar \& Wireless Commun., 2012, vol. 1, pp. 116-121, 3.

[18] S.-H. Ding, Q. Li, Y.-D. Li, and Q. Wang, "Continuous-wave terahertz digital holography by use of a pyroelectric array camera," Opt. Lett., vol. 36, no. 11, pp. 1993-1995, 2011.

[19] B. A. Knyazev et al., "Real-time imaging using a high-power monochromatic terahertz source: Comparative description of imaging techniques with examples of application," J. Infrared Millim. THz, vol. 32 , no. 10 , pp. 1207-1222, 2011.

[20] M. Locatelli et al., "Real-time terahertz digital holography with a quantum cascade laser," Sci. Rep., vol. 5, 2015, Art. ID 13566.

[21] L. Rong et al., "Terahertz in-line digital holography of human hepatocellular carcinoma tissue," Sci. Rep., vol. 5, p. 8445, 2015.

[22] L. Rong et al., "Terahertz in-line digital holography of dragonfly hindwing: Amplitude and phase reconstruction at enhanced resolution by extrapolation," Opt. Exp., vol. 22, no. 14, 2014, Art. ID 17236.

[23] K. Xue, Q. Li, Y.-D. Li, and Q. Wang, "Continuous-wave terahertz in-line digital holography," Opt. Lett., vol. 37, no. 15, p. 3228, 2012.

[24] Q. Li, K. Xue, Y.-D. Li, and Q. Wang, "Experimental research on terahertz gabor inline digital holography of concealed objects," Appl. Opt., vol. 51, no. 29, p. 7052, 2012.

[25] E. Hack and P. Zolliker, "Terahertz holography for imaging amplitude and phase objects," Opt. Exp., vol. 22, no. 13, 2014, Art. ID 16079.

[26] P. Zolliker and E. Hack, "THz holography in reflection using a high resolution microbolometer array," Opt. Exp., vol. 23, no. 9, 2015, Art. ID 10957.

[27] Y. Y. Choporova, B. A. Knyazev, and M. S. Mitkov, "Classical holography in the terahertz range: Recording and reconstruction techniques," IEEE Trans. THz Sci. Technol., vol. 5, no. 5, pp. 836-844, May 2015.

[28] Y. Zhang, W. Zhou, X. Wang, Y. Cui, and W. Sun, "Terahertz digital holography," Strain, vol. 44, no. 5, pp. 380-385, 2008.

[29] S. Wang, B. Ferguson, D. Abbott, and X.-C. Zhang, "T-ray imaging and tomography," J. Biol. Phys., vol. 29, no. 2-3, pp. 247-256, 2003.

[30] A. A. Gorodetsky and V. G. Bespalov, "THz pulse time-domain holography," in Proc. SPIE, 2010, vol. 7601, Art. ID 760107.

[31] V. Bespalov and A. Gorodetsky, "Modeling of referenceless holographic recording and reconstruction of images by means of pulsed terahertz radiation," J. Opt. Technol., vol. 74, no. 11, pp. 745-749, 2007.

[32] J. F. Federici, B. Schulkin, F. Huang, D. Gary, R. Barat, F. Oliveira, and D. Zimdars, "THz imaging and sensing for security applications- $\mathrm{Ex}-$ plosives, weapons and drugs," Semicond. Sci. Technol., vol. 20, no. 7, pp. S266-S280, 2005.

[33] V. Bespalov, V. Krylov, S. Putilin, and D. Stasel'ko, "Lasing in the far IR spectral range under femtosecond optical excitation of the inas semiconductor in a magnetic field," Opt. Spectrosc., vol. 93, no. 1, pp. 148-152, 2002.

[34] D. H. Auston, K. P. Cheung, and P. R. Smith, "Picosecond photoconducting Hertzian dipoles," Appl. Phys. Lett., vol. 45, no. 3, pp. 284-286, 1984.

[35] Q. Wu, T. Hewitt, and X.-C. Zhang, "Two-dimensional electro-optic imaging of THz beams," Appl. Phys. Lett., vol. 69, no. 8, pp. 1026-1028, 1996.

[36] T. Yasui, K. Sawanaka, A. Ihara, E. Abraham, M. Hashimoto, and T. Araki, "Real-time terahertz color scanner for moving objects," Opt. Exp., vol. 16, no. 2, pp. 1208-1221, 2008.

[37] A. Gorodetsky, A. D. Koulouklidis, M. Massaouti, and S. Tzortzakis, "Physics of the conical broadband terahertz emission from two-color laser-induced plasma filaments," Phys. Rev. A, vol. 89, no. 3, pp. 1-6, 2014.

[38] N. Balbekin, M. Kulya, P. Rogov, and N. Petrov, "The modeling peculiarities of diffractive propagation of the broadband terahertz two-dimensional field," Phys. Procedia, vol. 73, pp. 49-53, 2015.

[39] N. V. Petrov, V. G. Bespalov, and M. V. Volkov, "Phase retrieval of $\mathrm{THz}$ radiation using set of $2 \mathrm{D}$ spatial intensity measurements with different wavelengths," in Proc. SPIE, 2012, vol. 8281, pp. 82 810J-82 $810 \mathrm{~J}$.

[40] Q. Li, S.-H. Ding, Y.-D. Li, K. Xue, and Q. Wang, "Research on reconstruction algorithms in $2.52 \mathrm{THz}$ off-axis digital holography," $J$. Infrared, Millimeter, THz Waves, vol. 33, no. 10, pp. 1039-1051, 2012.
[41] L. Zhang, H. Zhong, C. Deng, C. Zhang, and Y. Zhao, "Terahertz wave reference-free phase imaging for identification of explosives," Appl. Phys. Lett., vol. 92, no. 9, 2008, Art. ID 091117.

[42] M. Massaouti, C. Daskalaki, A. Gorodetsky, A. D. Koulouklidis, and S. Tzortzakis, "Detection of harmful residues in honey using terahertz time-domain spectroscopy," Appl. Spectrosc., vol. 67, no. 11, pp. 1264-1269, 2013.

[43] Y.-C. Lin and C.-J. Cheng, "Determining the refractive index profile of micro-optical elements using transflective digital holographic microscopy," J. Opt., vol. 12, no. 11, 2010, Art. ID 115402.

[44] B. S.-Y. Ung, J. Li, H. Lin, B. M. Fischer, W. Withayachumnankul, and D. Abbott, "Dual-mode terahertz time-domain spectroscopy system," IEEE Trans. Terahertz Sci. Technol., vol. 3, no. 2, pp. 216-220, Jul. 2013.

[45] A. A. Gorodetsky and V. G. Bespalov, "THz computational holography process and optimization," in Proc. SPIE Integr. Optoelectron. Devices, 2008, vol. 6893, pp. 68 930F-68 930F-9.

[46] N. V. Petrov, A. N. Galiaskarov, T. Y. Nikolaeva, and V. G. Bespalov, "The features of optimization of a phase retrieval technique in $\mathrm{THz}$ frequency range," in Proc. SPIE, 2012, vol. 8413, p. 84131T.

[47] N. V. Petrov, A. A. Gorodetsky, and V. G. Bespalov, "Holography and phase retrieval in terahertz imaging," in Proc. SPIE, 2013, vol. 8846, p. $88460 \mathrm{~S}$.

[48] N. V. Petrov, V. G. Bespalov, and A. Gorodetsky, "Terahertz pulsed time-domain holography and iterative phase retrieval methods," in Proc. Opt. Photon. Taiwan Int. Conf., 2013, p. SAT-S0405-O003.

[49] M. S. Kulya, N. V. Petrov, A. N. Tcypkin, and V. G. Bespalov, "Influence of raster scan parameters on the image quality for the THz phase imaging in collimated beam with a wide aperture,"J. Phys. Conf. Ser., vol. 536, 2014, Art. ID 012010.

[50] M. Naftaly and R. Dudley, "Methodologies for determining the dynamic ranges and signal-to-noise ratios of terahertz time-domain spectrometers," Opt. Lett., vol. 34, no. 8, p. 1213, 2009.

[51] A. Eskicioglu and P. Fisher, "Image quality measures and their performance," IEEE Trans. Commun., vol. 43, no. 12, pp. 2959-2965, Dec. 1995.

[52] G. Chen and Q. Li, "Markov chain Monte Carlo sampling based terahertz holography image denoising," Appl. Opt., vol. 54, no. 14, p. 4345, 2015.

[53] Y. V. Grachev, M. O. Osipova, A. V. Kuz'mina, and V. G. Bespalov, "Determining the working band of frequencies of a pulsed terahertz spectrometer," J. Opt. Technol., vol. 81, no. 8, p. 468, 2014.

[54] K. Lee and J. Ahn, "Single-pixel coherent diffraction imaging," Appl. Phys. Lett., vol. 97, no. 24, 2010, Art. ID 241101.

[55] D. N. Puzyrev and A. A. Drozdov, "Collimation and focusing of initially single-cycle paraxial optical beams," J. Phys. Conf. Ser., vol. 541, 2014, Art. ID 012065

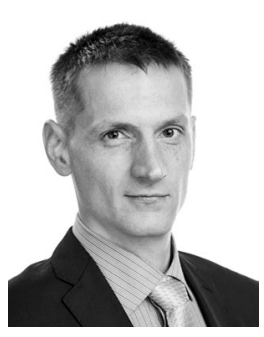

Nikolay V. Petrov received the B.Sc. and M.Sc. degrees in physics from Saint-Petersburg State University, St. Petersburg, Russia, in 2004 and 2007, respectively, and the Ph.D. degree in optics from the National Research University of Information Technology, Mechanics and Optics, St. Petersburg, Russia, in 2011.

Since 2011, he has been a Senior Researcher and Associate Professor with the Department of Photonics and Optical Information Technology, National Research University of Information Technology, Mechanics and Optics (ITMO University), St. Petersburg, Russia, where he leads the digital holography group. He has coauthored more than 30 journal papers and more than 100 conference proceedings. His research interests are currently focused on holography, femtosecond optics and terahertz imaging.

Dr. Petrov was the recipient of the Russian Federation Government Prize in Education in 2010 and is a winner of several prizes: St. Petersburg Youth Award in Education in 2012, Yu. I. Ostrovsky Second and First Prizes for the best scientific work in the field of optical holography and interferometry in 2012, and 2015 , respectively, and the St. Petersburg Government Prize in the field of scientific and pedagogical activity in 2015 . 


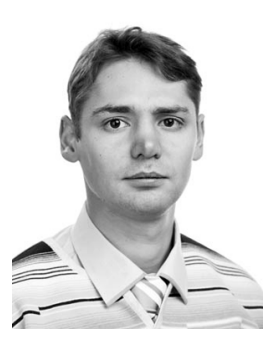

Maxim S. Kulya received the B.Sc., M.Sc. and $\mathrm{Ph} . \mathrm{D}$. degrees in photonics and optoinformatics from the National Research University of Information Technologies, Mechanics and Optics, Saint Petersburg, Russia, in 2008, 2010, and 2014, respectively.

$\mathrm{He}$ is currently working on the study of pulsed terahertz holography and phase imaging. Since 2010, he has been an Engineer with the International Laboratory of Femtosecond Optics and Femtotechnologies, National Research University of Information Technologies, Mechanics and Optics (ITMO University), St. Petersburg, Russia, , doing research work in the area of terahertz technologies.

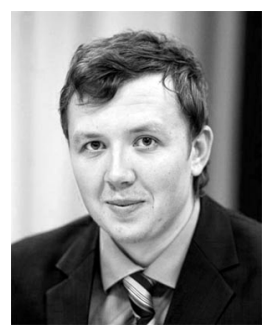

Anton N. Tsypkin received the B.Sc., M.Sc., and $\mathrm{Ph}$.D. degrees in photonics and optoinformatics from the National Research University of Information Technologies, Mechanics and Optics, St. Petersburg, Russia, in 2008, 2010, and 2013, respectively.

$\mathrm{He}$ is currently a head of THz Technologies Laboratory, Department of Photonics and Optical Information Technology and the International Laboratory of Femtosecond Optics and Femtotechnologies, National Research University of Information Technologies, Mechanics and Optics (ITMO University), St. Petersburg, Russia. His main scientific interests are $\mathrm{THz}$ imaging, $\mathrm{THz}$ spectroscopy and nonlinear optics in visible, IR and THz bands.

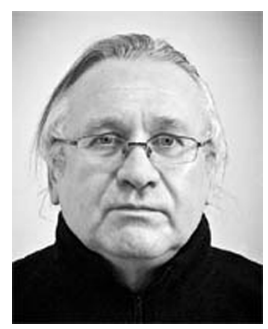

Victor G. Bespalov received the Ph.D. and Dr. Sci. degrees from the Vavilov State Optical Institute, St. Petersburg, Russia, in 1988 and 2003, respectively.

From 1978 to 2005, he was with the Vavilov State Optical Institute, St. Petersburg, Russia, first as a Researcher and later as a Principal Scientist. Since 2003, he has been a Professor with the Department of Photonics and Optical Informatics, National Research University of Information Technologies, Mechanics and Optics, St. Petersburg, Russia. $\mathrm{He}$ has authored and coauthored more than 120 articles in refereed journals and conference proceedings, including books and numerous invited talks at international conferences. He is laureate of Yu. I. Ostrovsky Prize for the best scientific work in the field of optical holography and interferometry in 2012. His current research interests include coherent and nonlinear optics, stimulated Raman scatterings, dynamic holography, femtosecond optics and $\mathrm{THz}$ imaging.

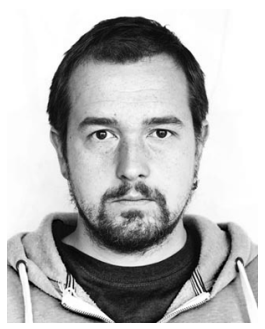

Andrei Gorodetsky received the B.Sc. and M.Sc. degrees in physics from Saint-Petersburg State University, St. Petersburg, Russia, in 2003 and 2006, respectively, and the Ph.D. degree in optics from the National Research University of Information Technologies, Mechanics and Optics, St. Petersburg, Russia, in 2010

Until 2011, he was with the Department of Photonics and Optical Informatics, National Research University of Information Technologies, Mechanics and Optics (ITMO University) as a Senior Researcher and an Assistant Professor. From 2011 to 2013, he was a Postdoctoral Researcher with the Ultrashort Non-linear Interactions and Sources group at the Institute of Electronic Structure and Laser, Foundation for Research and Technology-Hellas, Heraklion, Greece, where he was engaged in $\mathrm{THz}$ metamaterial research and controlled $\mathrm{THz}$ generation in two-color plasma filaments. Since 2014, he has been with the Optoelectronics and Biomedical Photonics group, Aston Institute of Photonic Technologies, Aston University, Birmingham, U.K. He is laureate of Yu. I. Ostrovsky Prize for the best scientific work in the field of optical holography and interferometry in 2012. His current research interests include $\mathrm{THz}$ imaging, pump-probe $\mathrm{THz}$ studies and development of quantum-dot-based compact $\mathrm{THz}$ sources. 